$2.168(45 \%)$, primaria completa 1.539 (32\%), secundaria incompleta $101(2 \%)$, secundaria completa $45(1 \%)$ y $2(0,04 \%)$ universitaria completa. Vivían en villas de emergencia 2.707 (56\%), en casas ocupadas 865 (18\%) y 1.247 (26\%) en casas de material. Por definición se consideró pobreza a la existencia de 2 de estas 3 variables: carencia alimentaria (por recibir una de dos comidas diarias adecuadas), carencia habitacional (por construcción precaria) y/o carencia educativa (analfabetos o con primaria incompleta). Así, en la población evaluada, se consideraron 3.572 pacientes (74\%) $(p<0,001)$ dentro del nivel de pobreza. Las conclusiones de este trabajo fueron contundentes:

1. En una población chagásica sana evaluada cardiológicamente por la Red de Chagas del GCBA, se observó un nivel significativo de desocupación por discriminación, que favorece la aparición de pobreza y bajo nivel educacional.

2. Los ocupados se encontraban "en negro" ( $\sin$ convenio de trabajo), por lo tanto, la gran desocupación sumada a esto último, determina la falta de acceso a una obra social, para una mejor calidad de atención médica y descuentos de medicación en casos de necesitarlos.

3. Los derechos al trabajo, estudio y vivienda digna se ven severamente vulnerados en una población "estigmatizada" por presentar Chagas a pesar de encontrarse sana para realizar todo tipo de actividad.

4. "La pobreza" en estos casos, puede ser causa o consecuencia de la enfermedad de Chagas, en cualquiera de estas dos formas posicionan a dichas personas en una situación de inferioridad grave ante el resto de la población no chagásica. La ética social parece no alcanzar a personas estigmatizadas por el solo hecho de tener un análisis de sangre reactivo.

Todo esto demuestra que será muy difícil en pocos años cambiar esta característica propia de la enfermedad como describe perfectamente el autor (1) con el criterio de "visibilidad e invisibilidad de la enfermedad", aún vigentes.

\section{REFERENCIAS BIBLIOGRÁFICAS}

1. Zabala JP. La enfermedad en su laberinto: avances, desafíos y paradojas de cien años del Chagas en Argentina. Salud Colectiva. 2012;8(Supl 1):S9-S21.

2. Auger SR, Storino R, De Rosa M, Caravello O, Gonzalez MI, Botaro E, Bonelli L, Rossini O. Chagas y sida, la importancia del diagnóstico precoz. Revista Argentina de Cardiología. 2005;73(6):439-445.

3. Storino R, Auger S, Jorg M. ¿Porqué la enfermedad de Chagas es un problema tan difícil de resolver? En: Manual práctico: Atención médica del paciente chagásico: un enfoque biológico, antropológico y social. Buenos Aires: Ediprof; 2003. p. 129-150.

4. Auger S, Gorocito M. Vías de Contagio. En: Enfermedad de Chagas: nuevo tratamiento de la cardiopatía incipiente. Sarrebruck: Editorial Academia Española; 2012. p. 7-14.

5. Auger S, Mitelman J, Giménez L, Abuin JC, Pérez Prado G, Vallaza M, Garro N, Caviglia S, et al. Red y Programa de Chagas del GCBA: Chagas y derechos humanos, aspectos médicos, psicosociales y éticos. Buenos Aires: $12^{\circ}$ Jornadas de Enfermedad de ChagasMazza: Chagas y Derechos Humanos, Aspectos médicos, psicosociales y éticos. (En prensa 2012).

\section{FORMA DE CITAR}

Auger S. Comentario sobre el criterio de visibilidad e invisibilidad en la enfermedad de Chagas-Mazza. [Debate]. Salud Colectiva. 2012;8(Supl 1):S29-S31.

\title{
Enfermedad de Chagas: un problema complejo
}

\section{Chagas disease: a complex problem}

\footnotetext{
Castro, Irma ${ }^{1}$

${ }^{1}$ Médica cardióloga. Ex jefe Diagnóstico y Tratamiento del Servicio Nacional de Chagas. Docente, Universidad Nacional de Córdoba, Argentina.irmacas@arnet.com.ar
}

Comentario sobre: Zabala JP. La enfermedad en su laberinto: avances, desafíos y paradojas de cien años del Chagas en Argentina. Salud Colectiva. 2012;8(Supl 1):S9-S21.

Coincido con Zabala (1) en que el Chagas es una entidad compleja, con factores biológicos, ambientales, económicos, laborales, políticos, de educación y socioculturales, cuya sumatoria conforma un círculo vicioso en el que 
el sujeto y su entorno están inmersos y del que difícilmente puedan salir.

La misma complejidad hace necesaria la intervención de equipos multidisciplinarios para el abordaje del problema en su integridad (2). También es cierto que, en el ámbito profesional médico, no se aprecia especial interés en el tema y se observan deficiencias cognitivas, como expresa el autor (1), posiblemente por déficit en su formación.

Se debe reconocer que las políticas sanitarias se han ocupado del problema, destinando recursos financieros muy elevados para el control, las viviendas, la promoción y la educación. Sin embargo, el problema subsiste como endemia en zonas donde no llegaron los recursos disponibles, zonas con alto riesgo de transmisión en los que no se han corregido los factores determinantes: se han realizado actividades de alto costo sin la continuidad indispensable para la sustentabilidad de los resultados.

Respecto de la investigación, se ha producido un gran desarrollo en las disciplinas que estudian el tema, con un gran impacto en el conocimiento del Trypanosoma cruzi, el vector, los mecanismos de transmisión, la inmunología, la patología, etc.; así se ha logrado una disminución de la transmisión, aunque no es general porque hay marcadas diferencias en algunas provincias.

El sujeto, víctima del problema, se invisibiliza por varios factores. Por un lado, la falta de conocimiento sobre la enfermedad, la baja demanda social, la disminución de expectativas, el aislamiento y abandono por carencia de información; por otro, la discriminación laboral, relacionada con el escaso grado de capacitación alcanzado y por la supuesta incapacidad laboral, dado que la admisión al trabajo termina con el resultado positivo de la serología, sin llegar a la definición de su estado clínico, lo que lo lleva a la frustración. De este modo se facilita un proceso que lo sumerge en el mercado de trabajo informal, con magros salarios y calidad de vida muy precaria, cerrándose así el círculo vicioso que no le permite progresar $(3,4)$.

La eficacia de las políticas sanitarias difiere en las provincias que mayor riesgo tienen, con estados de inercia para la prevención primaria y secundaria, actitud y aptitud que retroalimentan el problema, que se hace más notable a partir de la horizontalización de los programas de control.

Es evidente que el Chagas es un problema complejo que requiere:

- equipos multidisciplinarios altamente capacitados para la toma de decisiones, con recursos financieros y humanos que sean eficaces y eficientes;

- políticas nacionales articuladas con las provincias y las comunas en todas las regiones, con prioridad en las zonas de alto riesgo (5);

- continuidad política y administrativa para la aplicación de los programas;

- capacitación para el diagnóstico y tratamiento, para que los profesionales responsables sean protagonistas y no se comporten como observadores pasivos.

La urbanización del Chagas aumenta la complejidad asistencial por la asociación con otras patologías y estados de inmunodepresión, con alto costo social y económico del sistema sanitario, que requiere una masa crítica de profesionales para diagnosticar y tratar las reactivaciones.

Finalmente, la Ley 26.281 (6) obliga a la protección del sujeto, desde la prevención hasta el tratamiento gratuito, involucrando a todos los actores laborales, sociales y políticos; profesionales, técnicos o administrativos, con la responsabilidad que le corresponda a cada uno.

\section{REFERENCIAS BIBLIOGRÁFICAS}

1. Zabala JP. La enfermedad en su laberinto: avances, desafíos y paradojas de cien años del Chagas en Argentina. Salud Colectiva. 2012;8(Supl 1):S9-S21.

2. Pinto Dias JC. Evolución del conocimiento epidemiológico y situación epidemiológica actual. $1^{\circ}$ Congreso Virtual de Cardiología [Internet]. 2000 [citado 20 sep 2012]. Disponible en: http://www.fac.org.ar/cvirtual/cvirtesp/cientesp/ecesp/ecc4500c/cpinto/cpinto.htm

3. Briceño-León R. Enfermedad de Chagas: de la enfermedad rural a las epidemias urbanas. [Conferencia]. Segundas Jornadas de Fortalecimiento de la Enseñanza de Chagas en las Ciencias de la Salud. Córdoba, Universidad Nacional de Córdoba, octubre de 2010.

4. Briceño-León R, Gabaldón A. La casa enferma: sociología de la Enfermedad de Chagas. Caracas: Fondo Editorial Acta Científica Venezolana; 1990. 
5. Pinto Dias JC. La comunidad y el control de la enfermedad de Chagas: integración, rol, supervisión y evaluación de su participación [Internet]. Montevideo: Grupo de Trabajo OPS en Enfermedad de Chagas; 2001 [citado 20 sep 2012]. Disponible en: http://www.amro.who.int/spanish/ad/dpc/cd/consulta-7.pdf

6. Congreso de la Nación Argentina. Ley 26.281 [Internet] InfoLEG, Información Legislativa: 2007 [citado 20 sep
2012]. Disponible en: http://infoleg.mecon.gov.ar/ infolegInternet/anexos/130000-134999/131904/ norma.htm.

\section{FORMA DE CITAR}

Castro I. Enfermedad de Chagas: un problema complejo. [Debate]. Salud Colectiva. 2012;8(Supl 1):S31-S33.

\section{Enfermedad de Chagas: breve análisis de algunos factores que influyen en su persistencia}

\section{Chagas disease: A brief analysis of some of the factors influencing the disease's persistence}

\author{
Moretti, Edgardo ${ }^{1}$ \\ ${ }^{1}$ Doctor en Bioquímica. Especialista en Inmunología. Jefe de \\ Laboratorio, Servicio Nacional de Chagas. Profesor Adjunto, \\ Facultad de Ciencias Médicas, Universidad Nacional de \\ Córdoba, Argentina.eram112@live.com.ar
}

Comentario sobre: Zabala JP. La enfermedad en su laberinto: avances, desafíos y paradojas de cien años del Chagas en Argentina. Salud Colectiva. 2012;8(Supl 1):S9-S21.

La enfermedad de Chagas, como lo demuestra el excelente artículo del Dr. Juan Pablo Zabala (1), brinda un inmejorable ejemplo de la ineludible necesidad de encontrar puntos de conexión entre las ciencias biomédicas y las ciencias sociales, que a menudo transitan por carriles separados. La complejidad de esta endemia requiere del enfoque de distintas disciplinas, además de decisiones políticas a las cuales deberían aportar dichas disciplinas, de modo tal que los aspectos sociales, bioéticos, psicológicos, antropológicos y filosóficos no pueden estar ausentes en cualquier discusión que pretenda aportar no solo a hacer "visible" el problema, sino contribuir a su solución, siempre pensando en el hombre como objetivo central. Basten como ejemplo los conceptos de biopolítica y biopoder de Foucault (2) y la noción de cuerpos espectáculo, cuerpos esclavos y cuerpos con ideas de Badiou (3)
Abordaré algunos aspectos que considero trascendentes (en rigor todo el artículo lo es) desde una mirada biomédica por formación y social por inclinación personal.

Acuerdo con la lúcida forma de abordar el problema desde la tensión visibilidad-invisibilidad y me parece central la pregunta referida a la persistencia de la enfermedad durante más de 100 años y la crítica a las posturas simplistas que intentan explicarla.

Las múltiples paradojas que acompañan a la enfermedad de Chagas parten desde el descubrimiento del agente etiológico, el Trypanosoma cruzi y la enfermedad que el parásito produce. Por diversas circunstancias -entre ellas, el famoso "error de Carlos Chagas" al tratar de establecer una relación causa-efecto entre dos entidades como tripanosomiasis y bocio, asociadas en tiempo y espacio-, esta enfermedad siempre estuvo envuelta en discusiones que, más que contribuir a solucionar el problema, lo relegaron a un segundo plano supeditado a posiciones personales irreconciliables, vaivenes políticos, excesiva "medicalización", entre otras situaciones que favorecieron calificativos como "enfermedad olvidada", "endemia silenciosa y silenciada" o "mal escondido". Incluso la OMS la incluye entre las tristemente Ilamadas "enfermedades desatendidas", adjetivo que sin duda se aplica muy bien a los hombres y mujeres cuya "invisibilidad" los relega (1), como menciona Zabala, o como señalaba el Dr. Ramón Carrillo al expresar que: "Frente a las enfermedades que genera la miseria, frente a la tristeza, la angustia y el infortunio social de los pueblos, los microbios, como causas de enfermedad, son unas pobres causas".

En el artículo, se cita una publicación de la Organización Mundial de la Salud (OMS) (4) según la cual las "cifras oficiales" de infectados alcanzan a ocho millones. Si pocos años atrás en Latinoamérica ese número se estimaba 